\title{
Evaluación y selección de recursos web: una propuesta de calidad para la sección de referencia de la Biblioteca Universitaria de León
}

\author{
Evaluation al selection of web resources: a quality proposal for reference section \\ in the University Library of Leon
}

\section{Leticia BARrionueVo Almuzara, Lucía de Cos GonzÁlez-TALAdriz, María Marsá ViLA, Inmaculada BLANCO ALONSO y María del Mar GARCíA CASADO}

Biblioteca Universitaría de León, Universidad de León, León, España

\begin{abstract}
Resumen
Se propone un método de evaluación para medir la calidad de los recursos web de referencia (diccionarios y enciclopedias) que la Biblioteca Universitaria de León ofrece en su apartado "Recursos en Internet", dentro de la Biblioteca digital. El proyecto planteado por el Grupo de Trabajo de Recursos Electrónicos de la biblioteca, consiste en evaluar todos los recursos web incluidos en cada una de las categorías, siguiendo unos parámetros concretos, para determinar su grado de adecuación. Los resultados obtenidos tras la evaluación, nos ayudarán a seleccionar, entre la lista propuesta en un principio, aquellos materiales de referencia en formato electrónico, que sean más útiles para la comunidad universitaria, desechar aquellos que no alcancen la nota mínima exigida, y recomendar otros que cumplan al cien por cien los parámetros expuestos.
\end{abstract}

Palabras clave: Evaluación. Calidad. Recursos web. Biblioteca Universitaria de León. Sección de referencia.

\section{Introducción}

Entre los meses de febrero y marzo de 2007 la Biblioteca Universitaria de León elaboró su II Plan Estratégico, que fue aprobado por la Comisión General de Bibliotecas en el mes de mayo del mismo año. Con este II Plan la Biblioteca realizaba una nueva apuesta de futuro por la gestión de calidad para los años 2007/2009.

Conscientes de la importancia que para nuestros usuarios tenía la presentación y la organización de los recursos electrónicos en la página web de la Biblioteca, se constituyó un grupo de trabajo con la finalidad de desarrollar aspectos concretos de las líneas 4 (Servicios) y 5 (Colecciones) del Plan Estratégico. Dentro de sus actuaciones, se llevó a cabo una revisión en profundidad de la organización del apartado "Recursos electrónicos", comenzan-

\begin{abstract}
The purpose of this paper is to propose an evaluation method for measuring the quality of World Wide web reference resources (dictionaries and encyclopaedias) that Library of University of León make available through its Digital Library under the heading of "Resources on the internet". The project, undertaken by the Library Working Group on Electronic Resources, consists on evaluating all web resources included in each of the mentioned categories in order to determine their suitability. The results obtained after the quality assessment, will help us to select, among the list of proposals submitted at the beginning, those electronic reference resources that are more useful for the university community members, discarding those which fail to achieve the scoring proposed and recommending other that fulfill the parameters outlined.
\end{abstract}

Keywords: Evaluation. Quality. Web resources. Library of University of Leon. Reference Section.

do con las bases de datos y revistas electrónicas suscritas por la Biblioteca. Se facilitó el acceso a estos recursos mediante un sencillo buscador que permitiera la consulta por títulos y materias. Previamente, todas las bases de datos y revistas electrónicas se integraron en el catálogo de la Biblioteca habilitándose un acceso parcial a las mismas (scope).

Además de estos recursos adquiridos por compra, desde la página web de la Biblioteca se daba acceso a una selección de recursos de carácter gratuito disponibles en Internet. En este caso, la reestructuración consistió en la creación de dos apartados. Por un lado, el que denominamos "Guías temáticas", desde el que se ofrecía una selección de enlaces a recursos organizados por materias. Por otro, un apartado con acceso a información de tipo general, que llamamos "Recursos en Internet". La revisión y selección de los recursos temáticos se encomendó a los distintos 
responsables de las Bibliotecas de Área y el grupo de trabajo asumió la revisión y actualización del apartado destinado a los recursos de interés general.

Nuestro trabajo no partía de cero ya que se trataba de verificar que los recursos que ya formaban parte de la selección disponible en nuestra página web cumplían una serie de criterios de calidad, cuya definición sería el objetivo principal del trabajo del grupo. Estos recursos se habían venido introduciendo a lo largo del tiempo, sin criterios demasiado claros $y$, en muchos casos, hasta las URLs habian dejado de funcionar. En principio, se decidió mantener la división que de estos recursos generales se había hecho en la página web y comenzar la evaluación por el apartado "Diccionarios y enciclopedias". Los objetivos serían, por un lado, elaborar una herramienta de evaluación que sirviera para detectar la calidad de la selección de las obras de referencia que la Biblioteca ofrecía a sus usuarios desde la página web. En segundo lugar, hacer extensible la metodología de evaluación utilizada y aplicarla al resto de la selección de recursos disponibles. $Y$, en tercer lugar, sustituir aquellos recursos que no cumplieran los criterios de calidad mínima establecidos por otros que superaran la evaluación propuesta. Así, partiendo del apartado "Diccionarios y enciclopedias", se pretendía hacer una revisión profunda de la selección de "Recursos en Internet" y disponer de una herramienta de evaluación que nos permitiera seguir incorporando recursos web de calidad para nuestros usuarios.

\section{Marco teórico}

Uno de los objetivos que la biblioteca ha perseguido desde siempre es el de poner a disposición de sus usuarios fuentes de información para el estudio, propósito que sigue latente en la actualidad, a pesar de la vertiginosa evolución que han sufrido las bibliotecas en los últimos tiempos.

El rápido avance de la tecnología y la generalización del uso de Internet han favorecido el desarrollo de servicios electrónicos. Hoy en día, la mayoría de los portales web bibliotecarios incluyen un apartado dedicado a biblioteca digital, a partir del cual el usuario puede acceder a las fuentes y recursos en formato electrónico que la biblioteca propone. Bases de datos, revistas electrónicas, repositorios institucionales, revistas open access, o guías temáticas, son ejemplos de este tipo de recursos que se repite en la mayoría de los sitios web, ya que son los más solicitados por los usuarios.

Como ya adelantábamos en la introducción, nuestra evaluación comienza por los diccionarios y enciclopedias en línea, fuentes de conocimiento indispensables para el aprendizaje y la investigación.

2.1. Las obras de referencia en las bibliotecas: diccionarios y enciclopedias

Para García Ejarque (2000, p. 324) una "obra de referencia" es una "obra de consulta" y la define como aquella "hecha para buscar en ella información concreta según se necesita, pero no para leerla de cabo a rabo". Por su parte, Martín Vega (1995, p. 69-70) coincide en emplear obras de referencia como sinónimo de obras de consulta, añadiendo además que tradicionalmente han sido aquellas más asiduamente consultadas en cualquier biblioteca o centro de documentación. Según este autor, el concepto aparece por primera vez en 1871 y su uso se ha generalizado tanto que en muchas ocasiones sirve para designar cualquier clase de documento que cite al menos cincuenta referencias o noticias bibliográficas. Finalmente y siguiendo a Carrizo Sainero (1994, p. 37), "las obras de referencia o consulta" concentran en una sola publicación un número de noticias o informaciones tan amplio que su conocimiento detallado exigiría al lector la lectura de muchas páginas; de ahí que su elaboración sea compleja, debido a la necesidad de condensar los conocimientos, organizarlos y publicarlos de la formas más objetiva posible.

Dentro de este tipo de material podemos destacar distintas clases documentales: anuarios, atlas, bibliografías, guías, etc. pero los diccionarios y las enciclopedias son los ejemplos más abundantes en las secciones de referencia de las bibliotecas. La RAE (1) incluye dos acepciones del término "diccionario": por un lado, libro en el que se recogen y explican de forma ordenada voces de una o más lenguas, de una ciencia o de una materia determinada; y por otro, catálogo numeroso de noticias importantes de un mismo género, ordenado alfabéticamente.

Pons Serra (1999, p. 86-90) propone una clasificación de diccionarios atendiendo a siete puntos de vista diferentes: por la selección léxica del corpus, por el número de lenguas que incluyen, por la naturaleza de la información que proporcionan, por su carácter prescriptivo, por el nivel intelectual de sus contenidos, por la ordenación de los materiales, y por el soporte en que se presentan. En nuestro trabajo vamos a analizar diccionarios de carácter general, monolingües y multilin- 
gües, de definiciones y de sinónimos, con ordenación alfabética y en línea.

Según Cordón García (1999, p. 69-70) ,a pesar de que la historia de la enciclopedia se remonta a la antigüedad clásica, constituyendo un precipitado de conocimientos en los que se concitaba el saber de una época; actualmente, las enciclopedias se conciben como un conjunto de volúmenes en una disposición generalmente alfabética, elaboradas por equipos de especialistas, con abundante aparato bibliográfico e ilustraciones, e imbricadas orgánicamente por una red de referencias e índices.

\subsubsection{Evaluación y selección}

Fuentes Morán, García Palacios y Torres del Rey (2005, p. 75-78) consideran muy necesario desarrollar criterios objetivos que permitan delimitar los parámetros de medición de calidad de los diccionarios. Por ello, presentan tres ejes en los que se puede organizar toda evaluación de los mismos: cantidad, cantidad de los datos lexicográficos y usabilidad del diccionario. Estos autores muestran además una serie de preguntas relacionadas con estos criterios y a las que hay que dar respuesta en la evaluación: ¿Ofrece el diccionario suficientes datos? ¿Se puede encontrar fácil y rápidamente la información que se busca y que el diccionario contiene? ¿Son los datos inteligibles? ¿Permite el diccionario valorar su fiabilidad?

Por su parte, Cordón García (1999, p. 79-80) expone algunas de las preguntas que las enciclopedias de calidad deben responder: ¿La publica una editorial reconocida? ¿Escriben en ella especialistas en la materia? ¿Cuenta con una guía de uso? ¿Los índices están bien desarrollados? ¿Contiene un buen aparato de referencias cruzadas? ¿Las ilustraciones cumplen la función de complementar al texto?, ¿Cuenta con bibliografía actualizada y bien presentada? ¿Se actualiza convenientemente?

\subsection{Evaluación de recursos web: diccionarios y enciclopedias en la red}

Como señalan Jiménez Piano y Ortiz-Repiso (2007, p. 71) con la aparición de los documentos electrónicos ha surgido la necesidad de revisar los criterios tradicionales de evaluación. A pesar de existir poca homogeneidad en los estudios realizados sobre la evaluación de sedes web (2), todos cuentan con una estructura parecida: una lista de criterios que, a su vez, se desglosa en una serie de indicadores que los van acotando y que pueden constituir un cuestionario, incluyendo un modo de puntuación o quedar meramente expuestas.

Muchas han sido las instituciones y profesionales, sobre todo estadounidenses, que vinculados al ámbito científico han elaborado este tipo de listas. En nuestro país el estudio más importante es el de Lluís Codina (2000), que utiliza un método para evaluar sedes web basado en catorce indicadores, distribuidos en dos grupos: micronavegación o navegación interna y macronavegación. Además, organiza los indicadores en tres niveles, lo que significa que un recurso que llega al nivel 1 en un parámetro determinado alcanza la suficiencia, el nivel 2 sería notable, y el nivel 3 excelente.

En la línea de todos estos estudios, hemos trabajado; y el resultado es la lista de indicadores que hemos utilizado en nuestra investigación para evaluar los recursos de referencia que más adelante presentamos.

\section{Metodología}

Para conseguir los objetivos propuestos, se ha desarrollado una metodología que ha permitido aplicar una serie de herramientas, así como un sistema de medición necesario para indicar la calidad del recurso analizado.

La metodología se ha estructurado en varias etapas: establecimiento del objeto de estudio, elaboración del diseño metodológico y aplicación de un sistema de medición.

\subsection{Objeto de estudio}

El análisis se ha hecho sobre los recursos Web gratuitos (diccionarios e enciclopedias) seleccionados por la Biblioteca Universitaria y dispuestos en su página Web en el apartado:

Recursos electrónicos $>$ Recursos en Internet $>$ Diccionarios e Enciclopedias.

Un primer paso en la definición del objeto de estudio, consistió en valorar la pertinencia de los recursos sobre los que se iba a realizar el análisis, considerando si dicha muestra era objeto de estudio en su totalidad. Algunos recursos fueron descartados en primera instancia porque se consideró que no eran propiamente diccionarios o enciclopedias, y sí directorios u otro tipo de recursos.

Una vez definida la lista de recursos, se ha decido analizar todos y cada uno de los reflejados en la misma, estableciendo como premisa básica el no descartar ninguno a priori, ya que tomar la decisión de si son pertinentes o adecuados forma parte de los objetivos del trabajo planteado. 


\subsection{Desarrollo metodológico}

\subsubsection{Herramientas: parámetros e indicadores}

Se han definido ocho parámetros de evaluación teniendo en cuenta el tipo de recursos que se va a analizar y se engloban en dos bloques diferenciados: Diseño y Contenido.

- Dentro del diseño se han incluido los parámetros de accesibilidad, autodescripción, navegación y usabilidad.

- Dentro del contenido se han incluido los parámetros de autoría, actualidad, calidad y cantidad de información.

En cada parámetro se han estudiado y decidido un número de indicadores que ayudarán a concretar los criterios de evaluación establecidos por el grupo de trabajo, permitiendo definir el objeto de la evaluación, que no es otro que la calidad y pertinencia del recurso evaluado.

En la definición de los indicadores, se ha cuidado la formulación de las preguntas, para evitar contradicciones al valorar las respuestas. Así, por ejemplo, al preguntar ¿hay enlaces que no son funcionales?, si la respuesta es afirmativa y se le valora con un 3 , estamos fallando en el enunciado de la pregunta, pues se valoraría positivamente, cuando, en realidad, es algo negativo de cara a la evaluación del parámetro.

En la selección y evaluación de los indicadores, se han tenido en cuenta ciertos aspectos que se señalan a continuación.

Se ha considerado que tres indicadores del parámetro de navegación sólo son pertinentes para las enciclopedias por lo que se decide no utilizarlos para la evaluación de los diccionarios. Los indicadores son: ¿Dispone de un mapa Web o buscador que nos permita acceder a los contenidos sin tener que navegar? ¿Hay botones de enlace con la página siguiente y la anterior? ¿Está claramente indicada la ruta de navegación?

En el paramento de calidad y cantidad se ha descartado el indicador " ¿Tiene archivo histórico?", porque se consideró como no pertinente en este tipo de recursos. El indicador "¿Contiene información intrínsecamente valiosa?" se ha aplicado en los casos en los que las definiciones de los términos eran suficientemente explicativas, había definiciones desde distintos puntos de vista, o por el contrario, si éstas eran cortas y carecían de fundamento.

El indicador "¿Incluye información de valor añadido?" se ha aplicado en los diccionarios en el sentido de si ofrecía la pronunciación de la palabra, enlace a sinónimos o antónimos o aportaba información de palabras relacionadas.

En el parámetro de accesibilidad, al aplicar el indicador "¿La URL es estable?" se ha tenido en cuenta que ésta no se hubiera modificado desde que se colgó el recurso en la página web.

En el parámetro de autodescripción, se ha utilizado el indicador "¿Carece de errores de validación Web?" y, para analizar éste, se ha aplicado la herramienta del Consorcio Word Wide Web (W3C).

Finalmente, para medir el índice de popularidad se ha utilizado el Traffic-Rank de Alexa.

\subsubsection{Sistema de medición}

Para cada indicador, se han escogido cuatro opciones de calificación con los siguientes valores: 0 ) nunca / no; 1) poco aplicable / insuficiente; 2) bastante aplicable / suficiente; y 3 ) totalmente aplicable / bueno.

El motivo de elegir cuatro niveles de respuesta ha sido evitar la tendencia del evaluador a situarse en el punto central de calificación.

Cada parámetro tiene un peso específico (valor numérico) dentro de cada apartado, de manera que la suma de los valores de los parámetros que integran la evaluación es igual a 100. Cada apartado tiene definido un porcentaje o peso relativo de acuerdo a la importancia que desde el Grupo de trabajo se le ha dado dentro de la evaluación. Así, el bloque de diseño representa el $25 \%$ y el bloque del contenido el 75\% (tabla I).

\begin{tabular}{|c|c|c|c|}
\hline Parámetro & $\begin{array}{c}\text { Peso } \\
\text { específico }\end{array}$ & Bloque & $\begin{array}{c}\text { Peso } \\
\text { ponderado }\end{array}$ \\
\hline Autoría & 40 & \multirow[b]{3}{*}{ Contenido } & \multirow[b]{3}{*}{$75 \%$} \\
\hline Actualidad & 15 & & \\
\hline $\begin{array}{l}\text { Calidad y } \\
\text { cantidad de } \\
\text { información }\end{array}$ & 20 & & \\
\hline Accesibilidad & 7 & \multirow{4}{*}{ Diseño } & \multirow{4}{*}{$25 \%$} \\
\hline Autodescripción & 6 & & \\
\hline Navegación & 6 & & \\
\hline Usabilidad & 6 & & \\
\hline Total & 100 & Total & $100 \%$ \\
\hline
\end{tabular}

Tabla I. Parámetros y pesos

\subsection{Evaluación}

Partiendo de todo lo expuesto anteriormente, se ha procedido a evaluar cada recurso con los pasos que a continuación se detallan: 
Se ha elaborado una plantilla (3) por cada recurso a evaluar con los parámetros e indicadores seleccionados previamente. Cada parámetro contiene los indicadores que han ayudado a evaluar el recurso, los cuales, tal y como se exponía en el punto anterior, disponen de cuatro opciones de calificación. Asimismo, cada parámetro dispone de una casilla de evaluación total del mismo.

Cada recurso ha sido evaluado por todos los miembros del grupo, distribuyéndose los parámetros. Esta decisión parte de la consideración de que, de esa forma, cada parámetro será evaluado siempre desde un único punto de vista, eliminando una mayor subjetividad en el análisis y beneficiándose de la experiencia en su uso.

Una vez calificados todos los indicadores pertinentes, se ha procedido a evaluar cada parámetro, aplicando una formula de ponderación, que no es más que el resultado de la aplicación de una regla de tres simple: el valor máximo es al porcentaje máximo como el valor indicadores es a $\mathrm{X}$.
La formula consiste en hallar la suma de los valores de los indicadores de un mismo parámetro multiplicado por el porcentaje asignado al mismo y dividido por el valor máximo otorgado al parámetro.

Una vez obtenido el valor final de cada parámetro se han sumado todos los valores dando lugar al resultado final que es el que ha permitido aceptar o rechazar el recurso evaluado.

Hemos de destacar que, como punto de partida, se ha evaluado el Diccionario de la Real Academia de la Lengua Española, ya que el grupo de trabajo consideró que era el recurso que más se acercaba a obtener la calificación máxima y el que podía servir como referente en el apartado de los diccionarios.

\section{Resultados}

A la vista de los resultados obtenidos (figura 1), cabe hacer algunas consideraciones, como la de matizar la puntuación obtenida por algunos de los recursos.

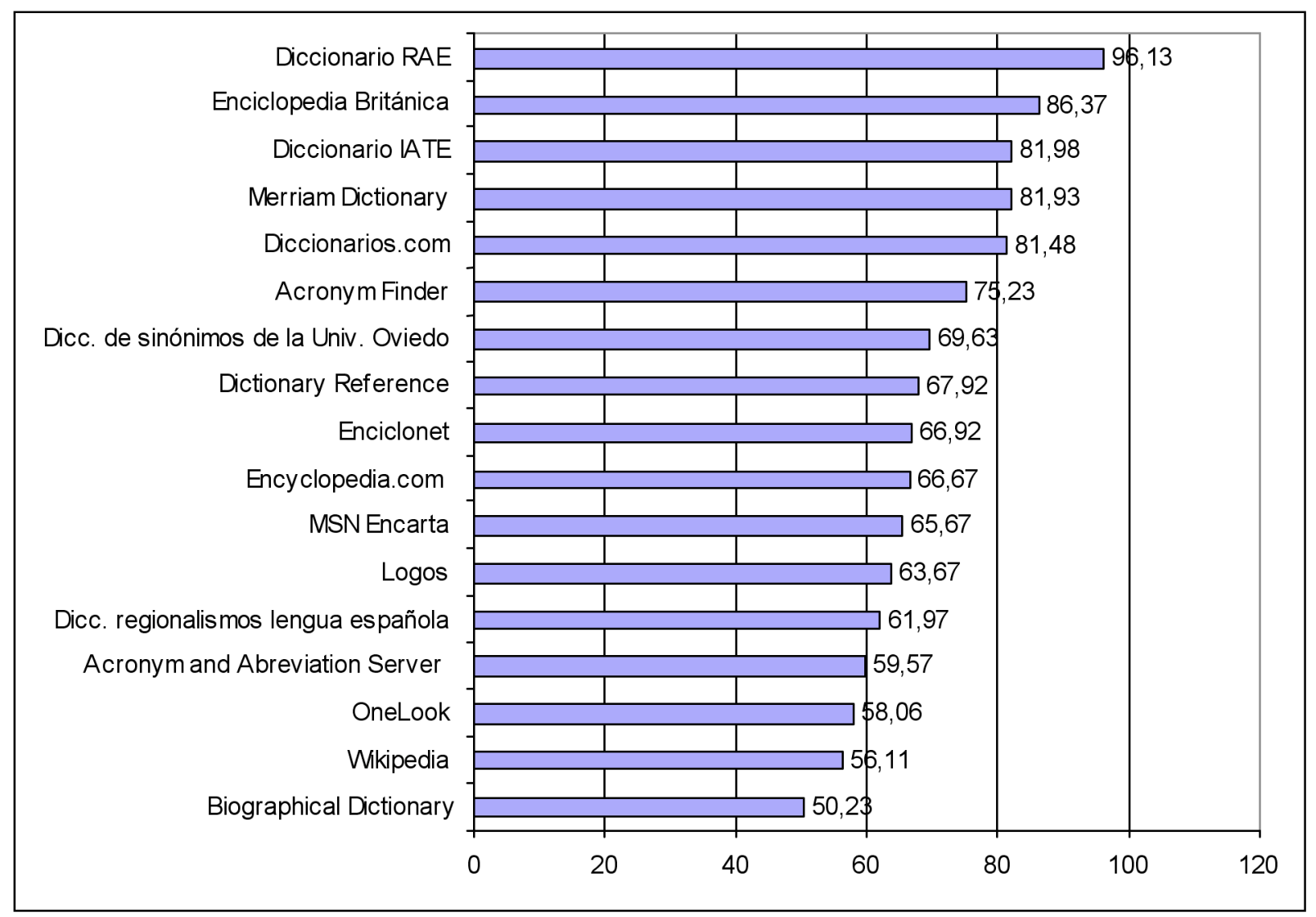

Figura 1. Resultados obtenidos 
Es el caso del portal diccionarios.com, cuyo acceso no es gratuito, sino promocional. Permite realizar cinco búsquedas en diferentes diccionarios en línea, pero limita el acceso a todos los demás servicios. En la evaluación del recurso, el parámetro de autoría ha alcanzado una puntuación muy alta $(37,77)$, lo cual ha compensado la baja puntuación obtenida por otros parámetros como accesibilidad $(3,5)$ y autodescripción $(3,25)$. Aunque el acceso limitado plantea dudas sobre su idoneidad como recurso puesto a disposición de los usuarios, la valoración del resto de los parámetros hace que sea considerado como recurso de calidad.

En el caso de la Enciclopedia Británica, que dispone de dos áreas de consulta, una para socios y otra de acceso gratuito, la parte que se ha evaluado ha sido la primera, ya que, como la Biblioteca posee la suscripción al recurso, los intentos de conectar con la versión libre fueron fallidos. A pesar de haber obtenido muy buen resultado $(86,37)$ en la evaluación, se plantea la idea de ubicar el recurso junto a los que la Biblioteca tiene suscritos ya que, como se indicó al principio, en el apartado "Recursos en Internet" sólo se incluyen los gratuitos.

Cabe destacar la baja puntuación global obtenida por un recurso tan popular como la Wikipedia. Si bien es cierto que algunos criterios como la cantidad y actualidad de la información, así como su accesibilidad y usabilidad, quedan fuera de toda duda, otros como la autoría y la información contrastada han provocado debates recientes entre la comunidad bibliotecaria. El elevado peso específico que el grupo de trabajo ha otorgado a la autoría $(40 \%$ del total) es el responsable de esta calificación final.

Lo mismo ocurre con el diccionario OneLook, que ha obtenido una calificación final baja respecto al resto de los recursos $(58,06)$. A pesar de ser un recurso aceptable y muy útil, la poca calificación es debida a que en la mayoría de parámetros tiene una calificación media-alta, pero muy baja en autoría.

Podemos concluir con la reflexión de que hay parámetros que quizás están excesivamente valorados, como puede ser el caso de la autoría; y, aunque los recursos evaluados han pasado todos por el sistema de evaluación obteniendo un "aprobado", quizás convenía revisar el peso específico de este parámetro de cara a futuras evaluaciones.

Como conclusión, podemos subrayar que se ha diseñado y puesto a punto una herramienta para la evaluación de los recursos electrónicos de libre acceso, que permitirá la inclusión en la página de "Recursos en Internet" únicamente de aquéllos que tengan una calidad contrastada y que superen los 50 puntos en la evaluación.

Además, ahora disponemos de un procedimiento sencillo y objetivo para aplicarlo a otros recursos electrónicos que no sean diccionarios o enciclopedias, sino otros tipos de fuentes de información. La herramienta creada podrá ser utilizada también por los bibliotecarios de las diferentes áreas, quienes tienen encargada la elaboración de guías por materias, en las que la inclusión de recursos electrónicos especializados es imprescindible, pero debe ser cuidadosamente estudiada.

Por último, hemos dado un paso más en nuestro proyecto de gestión de calidad, dirigido a ofrecer servicios acordes a las expectativas de profesores, investigadores y estudiantes. De este modo, las recomendaciones de la Biblioteca a sus usuarios estarán basadas en criterios de calidad y fiabilidad, y facilitarán su acceso a información de relevancia.

\section{Notas}

(1) Diccionario de la RAE.

(2) Apéndice I: lista de direcciones web, recopiladas por el Grupo de Trabajo, sobre la evaluación de recursos electrónicos (consulta: 27 mayo 2008)

(3) Apéndice II: plantilla utilizada para la evaluación.

(4) Apéndice III: gráfico que ilustra los resultados obtenidos del proceso de evaluación.

\section{Referencias.}

ALEXA. http://www.alexa.com/ (2008-05-14)

Ayuso García, Ma Dolores; Martínez Navarro, Victoria (2006). Evaluación de calidad de fuentes y recursos digitales: guía de buenas prácticas. // Anales de Documentación. 9 (2006). http://www.um.es/fccd/anales/ad09/ad0902.pdf (2008-04-02)

Carrizo, Gloria; Irureta-Goyena, Pilar; López de Quintana, Eugenio (1994). Manual de fuentes de información. Madrid: CEGAL, 1994.

Codina, Lluis (2000). El libro digital y la WWW. Madrid: Tauro Ediciones, 2000.

Cordón García, José Antonio (1999). Las enciclopedias. // Torres Ramírez, Isabel de (coord.). Las fuentes de información: estudios teórico-prácticos. Madrid: Síntesis, 1999. 65-80.

Diccionario de la RAE. http://www.rae.es/rae.html (2008-0428).

Diccionarios.com. http://www.diccionarios.com/ (2008-05-08).

Enciclopedia Británica. http://www.britannica.com/ (2008-0408).

Fernández Gómez, Sylvia (2006). Obras de referencia: enciclopedias, diccionarios, glosarios y otros recursos del idioma. // La información especializada en Internet. Madrid: CSIC, 2006. 173-194 
Fuentes Morán, Ma Teresa; García Palacios, Joaquín; Torres del Rey, Jesús (2005). Algunos apuntes sobre la evaluación de diccionarios. // Revista de Lexicografía. XI (2004-2005) 69-80.

García Ejarque, Luis (2000). Diccionario del archiverobibliotecario: terminología de la elaboración, tratamiento y utilización de los materiales propios de los centros documentales. Gijón: Trea, 2000.

Jiménez Piano, Marina; Ortiz-Repiso Jiménez (2007). Evaluación y calidad de sedes web. Gijon: Trea, 2007.

Martín Vega, Arturo (1995). Fuentes de información general. Gijón: Trea, 1995.
Martín Vega, Arturo (1997). La colección de referencia. II Boletín de la AAB. 13:48 (Septiembre 1997) 49-85.

OneLook Dictionary. http://www.onelook.com/ (2008-04-28).

Pons Serra, Amadeu (1999). Los diccionarios. // Torres Ramírez, Isabel de (coord.). Las fuentes de información: estudios teórico-prácticos. Madrid: Síntesis, 1999. 81-104.

W3C Markup Validation Service. http://validator.w3.org/ (200805-14

Wikipedia. http://es.wikipedia.org/wiki/Portada (2008-05-14).

\section{Apéndice I}

- http://www.loc.gov/rr/business/beonline/selectbib.html

- http://www.lib.vt.edu/help/instruct/evaluate/evalbiblio.html\#forms

- http://www.vuw.ac.nz/staff/alastair_smith/evaln/evaln.htm

- http://www.lib.vt.edu/help/instruct/evaluate/evaluating.html

- http://www.keele.ac.uk/depts/aa/landt/lt/Internet/criteria.htm

- http://www.ala.org/ala/acrl/acrlpubs/crlnews/backissues1998/julyaugust6/teachingundergrads.cfm

- http://www.vuw.ac.nz/staff/alastair_smith/evaln/index.htm

- http://www.library.ualberta.ca/instruction/science/evalweb.pdf

- http://www.library.cornell.edu/olinuris/ref/research/skill26.htm

- http://kathyschrock.com/abceval/index.htm

- http://www.library.cornell.edu/olinuris/ref/research/webeval.html

- http://www.library.ucla.edu/libraries/college/help/critical/index.htm

- http://mason.gmu.edu/ montecin/webcritique.htm

- http://www.ciolek.com/WWWVL-InfoQuality.html

- http://www.hopetillman.com/findqual.html

- http://www.oclc.org/research/projects/archive/wcp/pubs.htm

- http://www3.widener.edu/Academics/Libraries/Wolfgram_Memorial_Library/Evaluate_Web_Pages/659/

- http://liblearn.osu.edu/tutor/les1/

- http://www.robotwisdom.com/web/checklist.html

- http://lib.nmsu.edu/instruction/eval.html

- http://library.lib.binghamton.edu/instruct/classes/psyc473d.html\#criteria

- http://www.ciolek.com/WWWVLPages/QltyPages/QltyTruisms.html

- http://www.library.ualberta.ca/guides/criticalevaluation/index.cfm

- http://www.uflib.ufl.edu/admin/wwwtips.pdf

- http://www.lib.utah.edu/instruction/handouts/webeval.html

- http://www.virtualsalt.com/evalu8it.htm

- http://www.library.jhu.edu/researchhelp/general/evaluating/

- http://www.ncsu.edu/imse/3/evalweb.htm

- http://scout.wisc.edu/Reports/selection.php

- http://library.albany.edu/usered/eval/evalweb/

- http://www.alzado.org/articulo.php?id_art=75

- http://kathyschrock.net/abceval/

- http://lib.iastate.edu/commons/span390x/02spr/evaluar.html 


\section{Apéndice II}

Nombre del recurso:

Puntuación final

URL:

\begin{tabular}{|c|c|c|c|c|}
\hline \multicolumn{5}{|l|}{ AUTORIA } \\
\hline \multirow[t]{2}{*}{ Indicadores } & \multicolumn{4}{|c|}{ Calificación } \\
\hline & 0 & 1 & 2 & 3 \\
\hline \multicolumn{5}{|l|}{ ¿Está claro y explícito quién es el autor? } \\
\hline \multicolumn{5}{|l|}{ ¿Se indica la filiación profesional o institucional del autor? } \\
\hline \multicolumn{5}{|l|}{ ¿Tiene prestigio en la temática tratada? } \\
\hline \multicolumn{5}{|l|}{$\begin{array}{l}\text { ¿Se indica la formación y experiencia del autor en la materia? (otras } \\
\text { publicaciones...) }\end{array}$} \\
\hline \multicolumn{5}{|l|}{ ¿Se le cita con frecuencia en fuentes de calidad? } \\
\hline \multicolumn{5}{|l|}{$\begin{array}{l}\text { ¿Se ofrecen mecanismos para ponerse en contacto con el autor? (e-mail, } \\
\text { teléfono, dirección postal, fax...) }\end{array}$} \\
\hline Puntuación & & & & \\
\hline
\end{tabular}

\begin{tabular}{|l|c|c|c|c|}
\hline ACTUALIDAD & \multicolumn{5}{|c|}{ Calificación } \\
\hline Indicadores & 0 & 1 & 2 & 3 \\
\hline & & & & \\
\hline ¿Se actualiza periódicamente el recurso? & & & & \\
\hline ¿La mayoría de los enlaces son funcionales? & & & & \\
\hline ¿Están actualizados los enlaces? & & & \\
\hline Puntuación & & & \\
\hline
\end{tabular}

\begin{tabular}{|c|c|c|c|c|}
\hline \multicolumn{5}{|l|}{ CALIDAD Y CANTIDAD DE LA INFORMACIÓN } \\
\hline \multirow[t]{2}{*}{ Indicadores } & \multicolumn{4}{|c|}{ Calificación } \\
\hline & 0 & 1 & 2 & 3 \\
\hline \multicolumn{5}{|c|}{ ¿Hay algún indicio de que la información ha sido contrastada? } \\
\hline \multicolumn{5}{|c|}{ ¿Contiene información intrínsecamente valiosa? } \\
\hline \multicolumn{5}{|l|}{ ¿Incluye información de valor añadido? } \\
\hline \multicolumn{5}{|c|}{ ¿La información que contiene cubre los objetivos propuestos } \\
\hline \multicolumn{5}{|c|}{$\begin{array}{l}\text { ¿La cantidad de información que contiene el recurso es significativa en } \\
\text { comparación con otro de calidad contrastada? }\end{array}$} \\
\hline \multicolumn{5}{|c|}{ ¿Predomina la información útil sobre la trivial? } \\
\hline Puntuación & & & & \\
\hline
\end{tabular}

\begin{tabular}{|l|c|c|c|c|}
\hline ACCESIBILIDAD & \multicolumn{5}{|c|}{ Calificación } \\
\hline Indicadores & 0 & 1 & 2 & 3 \\
\hline & & & & \\
\hline ¿El recurso es gratuito? & & & & \\
\hline ¿Se puede acceder a todo el contenido sin necesidad de registrarse? & & & & \\
\hline ¿Se puede elegir el idioma de consulta de la interfaz? & & & & \\
\hline ¿Utiliza navegadores estándar (Internet Explorer, Mozilla Firefox y Opera? & & & & \\
\hline ¿Permite su consulta sin necesidad de instalar un software especifico? & & & & \\
\hline ¿El URL es estable? & & & \\
\hline Puntuación & \multicolumn{4}{|l}{} \\
\hline
\end{tabular}




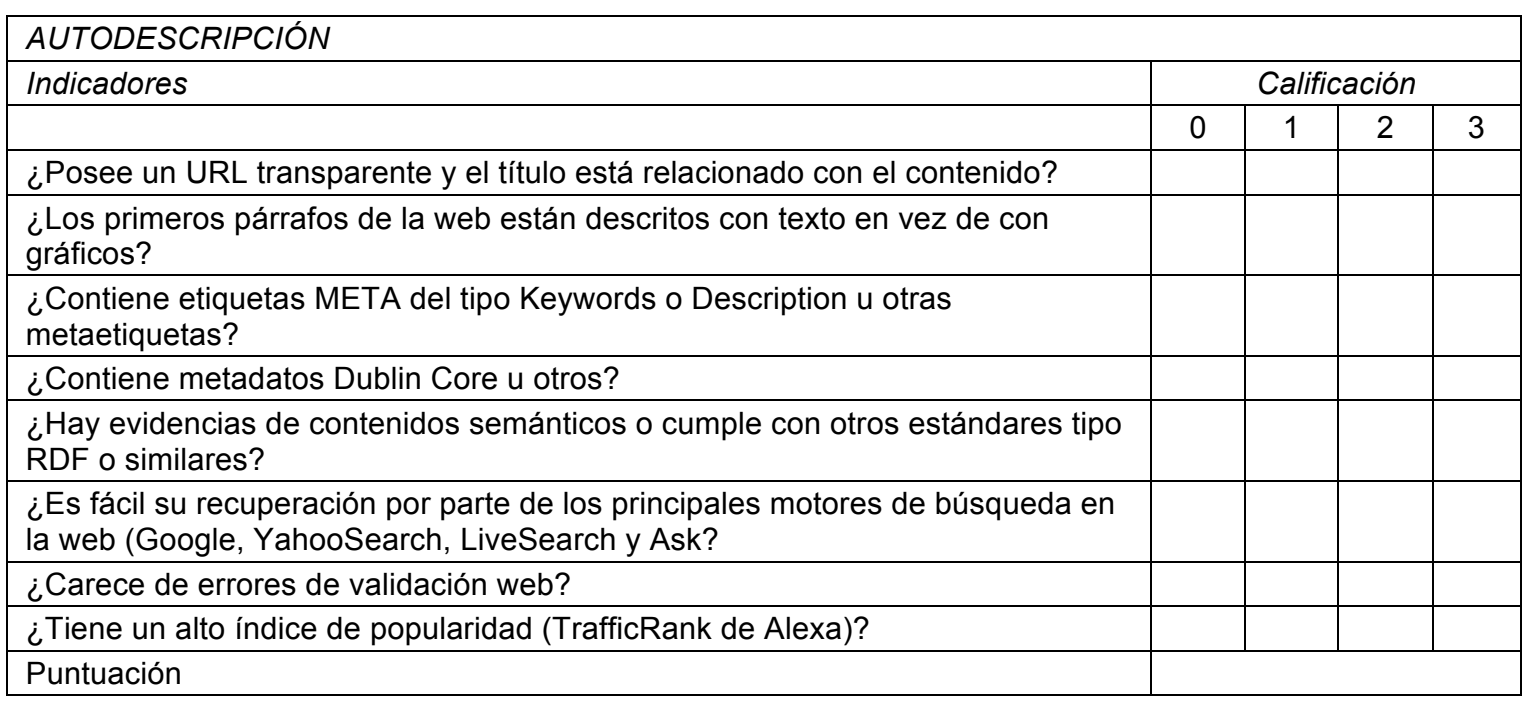

\begin{tabular}{|c|c|c|c|c|}
\hline \multicolumn{5}{|l|}{ NAVEGACIÓN } \\
\hline \multirow{2}{*}{ Indicadores } & \multicolumn{4}{|c|}{ Calificación } \\
\hline & 0 & 1 & 2 & 3 \\
\hline \multicolumn{5}{|l|}{ ¿Es fácil navegar por el recurso? } \\
\hline \multicolumn{5}{|l|}{$\begin{array}{l}\text { ¿Hay botones de enlace a la página de inicio y a otros sitios clave dentro del } \\
\text { recurso? }\end{array}$} \\
\hline \multicolumn{5}{|l|}{ ¿Cuenta con un buscador propio? } \\
\hline \multicolumn{5}{|l|}{$\begin{array}{l}\text { ¿Está claro el significado de los iconos o imágenes utilizados en el sistema de } \\
\text { navegación? }\end{array}$} \\
\hline Puntuación & & & & \\
\hline
\end{tabular}

\begin{tabular}{|c|c|c|c|c|}
\hline \multicolumn{5}{|l|}{ USABILIDAD } \\
\hline \multirow[t]{2}{*}{ Indicadores } & \multicolumn{4}{|c|}{ Calificación } \\
\hline & 0 & 1 & 2 & 3 \\
\hline \multicolumn{5}{|l|}{$\begin{array}{l}\text { ¿El contenido de la página está estructurado y organizado de forma intuitiva y } \\
\text { esta organización es fácilmente percibida por el usuario? }\end{array}$} \\
\hline \multicolumn{5}{|l|}{ ¿Hay un apartado de ayuda al usuario o permite la descarga de tutoriales? } \\
\hline \multicolumn{5}{|l|}{$\begin{array}{l}\text { ¿El diseño está al servicio del contenido, es atractivo y se mantiene uniforme a } \\
\text { lo largo de la página? }\end{array}$} \\
\hline \multicolumn{5}{|l|}{$\begin{array}{l}\text { ¿Se utiliza correctamente el color y la tipografía para poner de relieve el } \\
\text { contenido? }\end{array}$} \\
\hline \multicolumn{5}{|l|}{ ¿Permite descargar resultados de búsqueda o tiene una opción para imprimir? } \\
\hline Puntuación & & & & \\
\hline
\end{tabular}

